\title{
APPLICATIONS OF CERTAIN FUNCTIONS ASSOCIATED WITH LEMNISCATE BERNOULLI
}

\author{
Suzeini Abdul Halim ${ }^{1}$ And Rashidah OmaR ${ }^{2}$ \\ ${ }^{1,2}$ Institute of Mathematical Sciences, \\ Faculty of Science, University of Malaya, Malaysia, \\ suzeini@um.edu.my \\ ${ }^{2}$ Faculty of Computer and Mathematical Sciences, \\ MARA University of Technology Malaysia, \\ Ashidah@hotmail.com
}

\begin{abstract}
For $J(\alpha, f(z))=(1-\alpha) \frac{z f^{\prime}(z)}{f(z)}+\alpha\left[1+\frac{z f^{\prime \prime}(z)}{f^{\prime}(z)}\right] \quad(\alpha \geq 0)$, denote $\mathcal{S} \mathcal{L}(\alpha)$ and $\mathcal{S L}^{c}$ as classes of $\alpha$-convex and convex functions which respectively satisfy conditions $\left|[J(\alpha, f(z))]^{2}-1\right|<1$ and $\left|\left[1+\frac{z f^{\prime \prime}(z)}{f^{\prime}(z)}\right]^{2}-1\right|<1$. Using established results, namely $1+\beta z p^{\prime}(z) \prec \frac{1+D z}{1+E z}, 1+\frac{\beta z p^{\prime}(z)}{p(z)} \prec \frac{1+D z}{1+E z}$ and $1+\frac{\beta z p^{\prime}(z)}{p^{2}(z)} \prec \frac{1+D z}{1+E z}$ imply $p(z) \prec \sqrt{1+z}$ where $p(z)$ is an analytic function defined on the open unit disk $\mathbf{D}$ with $p(0)=1$. This article obtains conditions so that analytic functions $f$ belong to the classes $\mathcal{S} \mathcal{L}(\alpha)$ and $\mathcal{S} \mathcal{L}^{c}$.
\end{abstract}

Key words: Convex functions, differential subordination, lemniscate Bernoulli.

Abstrak. Untuk $J(\alpha, f(z))=(1-\alpha) \frac{z f^{\prime}(z)}{f(z)}+\alpha\left[1+\frac{z f^{\prime \prime}(z)}{f^{\prime}(z)}\right] \quad(\alpha \geq 0)$, nyatakan $\mathcal{S L}(\alpha)$ dan $\mathcal{S L}^{c}$ sebagai kelas-kelas dari konveks- $\alpha$ and fungsi-fungsi konveks yang secaraberturut-turut memenuhi kondisi $\left|[J(\alpha, f(z))]^{2}-1\right|<1$ dan $\left|\left[1+\frac{z f^{\prime \prime}(z)}{f^{\prime}(z)}\right]^{2}-1\right|<1$. Dengan menggunakan hasil-hasil yang telah diperoleh sebelumnya, yaitu $1+\beta z p^{\prime}(z) \prec \frac{1+D z}{1+E z}, 1+\frac{\beta z p^{\prime}(z)}{p(z)} \prec \frac{1+D z}{1+E z}$ dan $1+\frac{\beta z p^{\prime}(z)}{p^{2}(z)} \prec \frac{1+D z}{1+E z}$ mengakibatkan $p(z) \prec \sqrt{1+z}$ dimana $p(z)$ adalah sebuah fungsi analitis yang didefinisikan pada cakram buka $\mathbf{D}$ dengan $p(0)=1$. Artikel ini memperoleh kondisikondisi sehingga fungsi-fungsi analitis $f$ berada dalam kelas-kelas $\mathcal{S L}(\alpha)$ dan $\mathcal{S} \mathcal{L}^{c}$.

Kata kunci: Fungsi Konveks, subordinasi diferensial, lemniscate Bernoulli.

2000 Mathematics Subject Classification: 30C45, 30C50. 


\section{Introduction}

Let $\mathcal{A}$ denote the class of all analytic functions $f$ in the open unit disk $\mathbf{D}:=$ $\{z \in \mathbf{C}:|z|<1\}$ and normalised by $f(0)=0, f^{\prime}(0)=1$. An analytic function $f$ is subordinate to an analytic function $\mathrm{g}$, written $f(z) \prec g(z)(z \in \mathbf{D})$, if there exists an analytic function $w$ in $\mathbf{D}$ such that $w(0)=0$ and $|w(z)|<1$ for $|z|<1$ and $f(z)=g(w(z))$. In particular, if $g$ is univalent in $\mathbf{D}$, then $f(z) \prec g(z)$ is equivalent to $f(0)=g(0)$ and $f(\mathbf{D}) \subset g(\mathbf{D})$. For $f$ in $\mathcal{A}$ and $z \in \mathbf{D}$, the classes of starlike $S^{\star}$, convex $C$ and $\alpha$-convex functions are defined respectively by the conditions

$$
\begin{gathered}
S^{\star}=\left\{z \in \mathbf{D}: \mathfrak{R e} \frac{z f^{\prime}(z)}{f(z)}>0\right\} \\
C=\left\{z \in \mathbf{D}: \mathfrak{R e} \frac{z f^{\prime \prime}(z)}{f^{\prime}(z)}>-1\right\} \\
\mathcal{M}_{\alpha}=\{z \in \mathbf{D}: \mathfrak{R e}[J(\alpha, f(z))]>0\}
\end{gathered}
$$

where $J(\alpha, f(z))=(1-\alpha) \frac{z f^{\prime}(z)}{f(z)}+\alpha\left[1+\frac{z f^{\prime \prime}(z)}{f^{\prime}(z)}\right], \alpha \geq 0$. Note that $\mathcal{M}_{0}=S^{\star}$ and $\mathcal{M}_{1}=C$.

Sokól and Stankiewicz [5] introduced the class $S L^{\star}$ consisting of normalised analytic functions $f$ in $\mathbf{D}$ satisfying the condition $\left|\left[\frac{z f^{\prime}(z)}{f(z)}\right]^{2}-1\right|<1$ for $z \in \mathbf{D}$. Geometrically, a function $f \in S L^{\star}$ if $\frac{z f^{\prime}(z)}{f(z)}$ is in the interior of the right half of the lemniscate of Bernoulli $\left(x^{2}+y^{2}\right)^{2}-2\left(x^{2}-y^{2}\right)=0$. A function in the class $S L^{\star}$ is called a Sokól-Stankiewicz starlike function. Alternatively, we can also write $f \in S L^{\star} \Leftrightarrow \frac{z f^{\prime}(z)}{f(z)} \prec \sqrt{1+z}$. Some properties of functions in class $S L^{\star}$ have been studied by [1], [4], [6], [7], [8] and [9]. Next, we denote $S^{\star}[A, B]$ as the class of Janowski starlike functions defined in Janowski [2] consisting of functions $f \in \mathcal{A}$ satisfying $\frac{z f^{\prime}(z)}{f(z)} \prec \frac{1+A z}{1+B z}(-1 \leq B<A \leq 1)$.

Let $\mathcal{S} \mathcal{L}(\alpha)$ and $\mathcal{S} \mathcal{L}^{c}$ denote the classes of $\alpha$-convex and convex functions which respectively satisfy $\left|[J(\alpha, f(z))]^{2}-1\right|<1$ and $\left|\left[1+\frac{z f^{\prime \prime}(z)}{f^{\prime}(z)}\right]^{2}-1\right|<1(z \in$ D). It is obvious that $f \in \mathcal{S L}(\alpha) \Leftrightarrow J(\alpha, f(z)) \prec \sqrt{1+z}$ and $f \in \mathcal{S L}^{c} \Leftrightarrow$ $1+\frac{z f^{\prime \prime}(z)}{f^{\prime}(z)} \prec \sqrt{1+z}$. Recently in [4] the authors determined condition on $\beta$ so that $1+\beta z p^{\prime}(z), 1+\frac{\beta z p^{\prime}(z)}{p(z)}$ and $1+\frac{\beta z p^{\prime}(z)}{p^{2}(z)}$ are subordinated to $\frac{1+D z}{1+E z}$ imply $p(z)$ is subordinated to $\sqrt{1+z}$ where $p(z)$ is analytic in $\mathbf{D}$ with $p(0)=1$. Properties of functions in the class $\mathcal{S} \mathcal{L}(\alpha)$ and $\mathcal{S} \mathcal{L}^{c}$ are obtained using results given in [4]. In proving the lemmas, the following proposition is needed.

Proposition 1.1. [3] Let $q$ be univalent in $\boldsymbol{D}$ and let $\varphi$ be analytic in a domain containing $q(\boldsymbol{D})$. Let $z q^{\prime}(z) \varphi[q(z)]$ be starlike. If $p$ is analytic in $D, p(0)=q(0)$ and satisfies $z p^{\prime}(z) \varphi[p(z)] \prec z q^{\prime}(z) \varphi[q(z)]$ then $p \prec q$ and $q$ is the best dominant. 
Given that $|E|<1,|D| \leq 1$ and $D \neq E$, we then have the following lemmas.

Lemma 1.2. Let $\beta_{0}=\frac{2 \sqrt{2}|D-E|}{(1-|E|)}$. If $1+\beta z p^{\prime}(z) \prec \frac{1+D z}{1+E z}\left(\beta \geq \beta_{0}\right)$ then $p(z) \prec$ $\sqrt{1+z}$.

Proof. By letting $q(z)=\sqrt{1+z}$ and using Proposition 1.1, it suffices to show

$$
s(z)=\frac{1+D z}{1+E z} \prec 1+\beta z q^{\prime}(z)=1+\frac{\beta z}{2 \sqrt{1+z}}=h(z) .
$$

Quite straightforward it can be established that $\left|s^{-1}[h(z)]\right| \geq 1$ for $\beta \geq \frac{2 \sqrt{2}|(D-E)|}{(1-|E|)}$. Thus the result.

Lemma 1.3. Let $\beta_{0}=\frac{4|D-E|}{(1-|E|)}$. If $1+\beta \frac{z p^{\prime}(z)}{p(z)} \prec \frac{1+D z}{1+E z}\left(\beta \geq \beta_{0}\right)$ then $p(z) \prec \sqrt{1+z}$.

Lemma 1.4. Let $\beta_{0}=\frac{4 \sqrt{2}|D-E|}{(1-|E|)}$. If $1+\beta \frac{z p^{\prime}(z)}{p^{2}(z)}\left(\beta \geq \beta_{0}\right) \prec \frac{1+D z}{1+E z}$ then $p(z) \prec$ $\sqrt{1+z}$.

With appropriate choices of $\varphi$ in Proposition 1.1, and using similar approach as above Lemma 1.3 and Lemma 1.4 is easily verified. Details of proving these lemmas can be found in [4].

\section{Main Results}

Theorem 2.1. Let $\beta_{0}=\frac{2 \sqrt{2}(|D-E|)}{1-|E|},|E|<1,|D| \leq 1, D \neq E, \beta \geq \beta_{0}$ and $f \in A$. If $f$ satisfies

$1+\beta\left\{(1-\alpha) \frac{z f^{\prime}(z)}{f(z)}\left(\frac{z f^{\prime \prime}(z)}{f^{\prime}(z)}-\frac{z f^{\prime}(z)}{f(z)}+1\right)+\alpha \frac{z f^{\prime \prime}(z)}{f^{\prime}(z)}\left(\frac{z\left[f^{\prime \prime}(z)\right]^{\prime}}{f^{\prime \prime}(z)}-\frac{z f^{\prime \prime}(z)}{f^{\prime}(z)}+1\right)\right\} \prec \frac{1+D z}{1+E z}$

then $f \in \mathcal{S} \mathcal{L}(\alpha)$

Proof. With $p(z)=(1-\alpha)\left[\frac{z f^{\prime}(z)}{f(z)}\right]+\alpha\left[1+\frac{z f^{\prime \prime}(z)}{f^{\prime}(z)}\right]$, we have

$$
\begin{aligned}
p^{\prime}(z) & =(1-\alpha)\left\{\frac{f(z)\left[z f^{\prime \prime}(z)+f^{\prime}(z)\right]-z\left[f^{\prime}(z)\right]^{2}}{[f(z)]^{2}}\right\}+\alpha\left\{\frac{f^{\prime}(z)\left[z\left(f^{\prime \prime}(z)\right)^{\prime}+f^{\prime \prime}(z)\right]-z\left[f^{\prime \prime}(z)\right]^{2}}{\left[f^{\prime}(z)\right]^{2}}\right\} \\
& =(1-\alpha) \frac{f^{\prime}(z)}{f(z)}\left\{\frac{z f^{\prime \prime}(z)}{f^{\prime}(z)}+1-\frac{z f^{\prime}(z)}{f(z)}\right\}+\alpha \frac{f^{\prime \prime}(z)}{f^{\prime}(z)}\left\{\frac{z\left[f^{\prime \prime}(z)\right]^{\prime}}{f^{\prime \prime}(z)}+1-\frac{z f^{\prime \prime}(z)}{f^{\prime}(z)}\right\}
\end{aligned}
$$

and from Lemma 1.2, the result implies $f \in \mathcal{S} \mathcal{L}(\alpha)$.

For the special case $\alpha=1$, Theorem 2.1 gives the result for the class $\mathcal{S} \mathcal{L}^{c}$. 
Corollary 2.2. Let $\beta_{0}=\frac{2 \sqrt{2}(|D-E|)}{1-|E|},|E|<1,|D| \leq 1, D \neq E, \beta \geq \beta_{0}$ and $f \in A$. If $f$ satisfies

$$
1+\beta \frac{z f^{\prime \prime}(z)}{f^{\prime}(z)}\left\{\frac{z\left[f^{\prime \prime}(z)\right]^{\prime}}{f^{\prime \prime}(z)}-\frac{z f^{\prime \prime}(z)}{f^{\prime}(z)}+1\right\} \prec \frac{1+D z}{1+E z}
$$

then $f \in \mathcal{S L}^{c}$.

ProOF. With $p(z)=1+\frac{z f^{\prime \prime}(z)}{f^{\prime}(z)}$, it follows that

$$
\begin{aligned}
z p^{\prime}(z) & =\frac{z^{2}\left[f^{\prime \prime}(z)\right]^{\prime}}{f^{\prime}(z)}+\frac{z f^{\prime \prime}(z)}{f^{\prime}(z)}-\frac{z^{2}\left[f^{\prime \prime}(z)\right]^{2}}{\left[f^{\prime}(z)\right]^{2}} \\
& =\frac{z f^{\prime \prime}(z)}{f^{\prime}(z)}\left\{\frac{z\left[f^{\prime \prime}(z)\right]^{\prime}}{f^{\prime \prime}(z)}+1-\frac{z f^{\prime \prime}(z)}{f^{\prime}(z)}\right\},
\end{aligned}
$$

and applying Lemma 1.2 gives $1+\frac{z f^{\prime \prime}(z)}{f^{\prime}(z)} \prec \frac{1+D z}{1+E z}$, hence $f \in \mathcal{S L}^{c}$.

Theorem 2.3. Let $\beta_{0}=\frac{4|D-E|}{(1-|E|)}$ and $\beta \geq \beta_{0}$. Suppose $f \in A$ and

$$
1+\beta\left\{\frac{(1-\alpha) z f^{\prime}(z)\left[1-\frac{z f^{\prime}(z)}{f(z)}+\frac{z f^{\prime \prime}(z)}{f^{\prime}(z)}\right]}{(1-\alpha) z f^{\prime}(z)+\alpha f(z)\left[1+\frac{z f^{\prime \prime}(z)}{f^{\prime}(z)}\right]}+\frac{\alpha z f^{\prime \prime}(z)\left[1-\frac{z f^{\prime \prime}(z)}{f^{\prime}(z)}+\frac{z\left[f^{\prime \prime}(z)\right]^{\prime}}{f^{\prime \prime}(z)}\right]}{(1-\alpha) f^{\prime}(z) \frac{z f^{\prime}(z)}{f(z)}+\alpha\left[f^{\prime}(z)+z f^{\prime \prime}(z)\right]}\right\} \prec \frac{1+D z}{1+E z}
$$

then $f \in \mathcal{S} \mathcal{L}(\alpha)$. 
Proof. Let $p(z)=(1-\alpha)\left[\frac{z f^{\prime}(z)}{f(z)}\right]+\alpha\left[1+\frac{z f^{\prime \prime}(z)}{f^{\prime}(z)}\right]$.

$$
\begin{aligned}
\frac{p^{\prime}(z)}{p(z)}= & \frac{(1-\alpha) \frac{f^{\prime}(z)}{f(z)}\left\{\frac{z f^{\prime \prime}(z)}{f^{\prime}(z)}+1-\frac{z f^{\prime}(z)}{f(z)}\right\}+\alpha \frac{f^{\prime \prime}(z)}{f^{\prime}(z)}\left\{\frac{z\left[f^{\prime \prime}(z)\right]^{\prime}(z)}{f^{\prime \prime}(z)}+1-\frac{z f^{\prime \prime}(z)}{f^{\prime}(z)}\right\}}{(1-\alpha)\left[\frac{z f^{\prime}(z)}{f(z)}\right]+\alpha\left[1+\frac{z f^{\prime \prime}(z)}{f^{\prime}(z)}\right]} \\
= & \frac{(1-\alpha) f^{\prime}(z)\left\{z f^{\prime \prime}(z)+f^{\prime}(z)-\frac{z f^{\prime}(z) f^{\prime}(z)}{f(z)}\right\}}{f^{\prime}(z)\left\{(1-\alpha) z f^{\prime}(z)+\alpha f(z)\left(1+\frac{z f^{\prime \prime}(z)}{f^{\prime}(z)}\right)\right\}}+\frac{\alpha f^{\prime \prime}(z) f(z)\left\{\frac{z\left[f^{\prime \prime}(z)\right]^{\prime}}{f^{\prime \prime}(z)}+1-\frac{z f^{\prime \prime}(z)}{f^{\prime}(z)}\right\}}{f(z)\left\{(1-\alpha) f^{\prime}(z) \frac{z f^{\prime}(z)}{f(z)}+\alpha\left[f^{\prime}(z)+z f^{\prime \prime}(z)\right]\right\}} \\
= & \frac{(1-\alpha) f^{\prime}(z)\left\{\frac{z f^{\prime \prime}(z)}{f^{\prime}(z)}+\frac{f^{\prime}(z)}{f^{\prime}(z)}-\frac{z\left[f^{\prime}(z)\right]^{2}}{f^{\prime}(z) f(z)}\right\}}{(1-\alpha) z f^{\prime}(z)+\alpha f(z)\left[1+\frac{z f^{\prime \prime}(z)}{f^{\prime}(z)}\right]} \frac{\alpha f^{\prime \prime}(z) f(z)}{f(z)}\left\{\frac{z\left[f^{\prime \prime}(z)\right]^{\prime}}{f^{\prime \prime}(z)}+1-\frac{z f^{\prime \prime}(z)}{f^{\prime}(z)}\right\} \\
= & \frac{(1-\alpha) f^{\prime}(z) \frac{z f^{\prime}(z)}{f(z)}+\alpha\left[f^{\prime}(z)+z f^{\prime \prime}(z)\right]}{(1-\alpha) z f^{\prime}(z)+\alpha f(z)\left[1+\frac{z f^{\prime \prime}(z)}{f^{\prime}(z)}\right]}+\frac{\alpha f^{\prime \prime}(z)\left\{\frac{z\left[f^{\prime \prime}(z)\right]^{\prime}}{f^{\prime \prime}(z)}+1-\frac{z f^{\prime \prime}(z)}{f^{\prime}(z)}\right\}}{(1-\alpha) f^{\prime}(z) \frac{z f^{\prime}(z)}{f(z)}+\alpha\left[f^{\prime}(z)+z f^{\prime \prime}(z)\right]}
\end{aligned}
$$

Applying Lemma 1.3 gives $f \in \mathcal{S} \mathcal{L}(\alpha)$.

For $\alpha=1$ we have the result for the class $\mathcal{S} \mathcal{L}^{c}$.

Corollary 2.4. Let $\beta_{0}=\frac{4|D-E|}{(1-|E|)}, \beta \geq \beta_{0}$ and $f \in A$. If

$$
1+\beta \frac{z f^{\prime \prime}(z)}{\left[f^{\prime}(z)+z f^{\prime \prime}(z)\right]}\left\{\frac{z\left[f^{\prime \prime}(z)\right]^{\prime}}{f^{\prime \prime}(z)}-\frac{z f^{\prime \prime}(z)}{f^{\prime}(z)}+1\right\} \prec \frac{1+D z}{1+E z}
$$

then $f \in \mathcal{S L}^{c}$.

Proof. For $p(z)=1+\frac{z f^{\prime \prime}(z)}{f^{\prime}(z)}$, hence

$$
\begin{aligned}
\frac{z p^{\prime}(z)}{p(z)} & =\frac{z f^{\prime \prime}(z)}{f^{\prime}(z)}\left[\frac{f^{\prime}(z)-z f^{\prime \prime}(z)}{f^{\prime}(z)}\right]\left[\frac{f^{\prime}(z)}{f^{\prime}(z)+z f^{\prime \prime}(z)}\right]+\left[\frac{z^{2}\left[f^{\prime \prime}(z)\right]^{\prime}}{f^{\prime}(z)}\right]\left[\frac{f^{\prime}(z)}{f^{\prime}(z)+z f^{\prime \prime}(z)}\right] \\
& =\frac{z f^{\prime \prime}(z)}{\left[f^{\prime}(z)+z f^{\prime \prime}(z)\right]}-\frac{\left[z f^{\prime \prime}(z)\right]^{2}}{f^{\prime}(z)\left[f^{\prime}(z)+z f^{\prime \prime}(z)\right]}+\frac{z^{2}\left[f^{\prime \prime}(z)\right]^{\prime}}{\left[f^{\prime}(z)+z f^{\prime \prime}(z)\right]} \\
& =\frac{z f^{\prime \prime}(z)}{\left[f^{\prime}(z)+z f^{\prime \prime}(z)\right]}\left\{\frac{z\left[f^{\prime \prime}(z)\right]^{\prime}}{f^{\prime \prime}(z)}+1-\frac{z f^{\prime \prime}(z)}{f^{\prime}(z)}\right\}
\end{aligned}
$$


and based on Lemma $1.3, f \in \mathcal{S L}^{c}$.

Theorem 2.5. Let $\beta_{0}=\frac{4 \sqrt{2}|D-E|}{(1-|E|)}, \beta \geq \beta_{0}$ and $f \in A$.

$$
\begin{gathered}
1+\beta z\left\{\frac{(1-\alpha)\left[f^{\prime}(z)\right]^{3} f(z)\left[\frac{z f^{\prime \prime}(z)}{f^{\prime}(z)}+1-\frac{z f^{\prime}(z)}{f(z)}\right]+\alpha[f(z)]^{2} f^{\prime}(z) f^{\prime \prime}(z)\left[\frac{z\left[f^{\prime \prime}(z)\right]^{\prime}}{f^{\prime \prime}(z)}+1-\frac{z f^{\prime \prime}(z)}{f^{\prime}(z)}\right]}{\left[(1-\alpha) f^{\prime}(z) z f^{\prime}(z)+\alpha f(z)\left[f^{\prime}(z)+z f^{\prime \prime}(z)\right]\right]^{2}}\right\} \\
\prec \frac{1+D z}{1+E z} \Rightarrow f \in \mathcal{S L}(\alpha) .
\end{gathered}
$$

Proof. Using Lemma 1.4 with $p(z)=(1-\alpha)\left[\frac{z f^{\prime}(z)}{f(z)}\right]+\alpha\left[1+\frac{z f^{\prime \prime}(z)}{f^{\prime}(z)}\right]$ gives the desired result.

Corollary 2.6. Let $\beta_{0}=\frac{4 \sqrt{2}|D-E|}{(1-|E|)}, \beta \geq \beta_{0}$ and $f \in A$.

$$
1+\beta \frac{z f^{\prime}(z) f^{\prime \prime}(z)}{\left[f^{\prime}(z)+z f^{\prime \prime}(z)\right]^{2}}\left\{\frac{z\left[f^{\prime \prime}(z)\right]^{\prime}}{f^{\prime \prime}(z)}+1-\frac{z f^{\prime \prime}(z)}{f^{\prime}(z)}\right\} \prec \frac{1+D z}{1+E z} \Rightarrow f \in \mathcal{S} \mathcal{L}^{c} .
$$

PROOF. Set $p(z)=1+\frac{z f^{\prime \prime}(z)}{f^{\prime}(z)}$ and in a similar manner the result is easily obtained.

\section{Concluding Remark}

For $\alpha=0$, Theorem 2.1, Theorem 2.3 and Theorem 2.5 reduce to the results for $f \in S L^{\star}$

Acknowledgement This research was supported by University of Malaya grants: UMRG108/10APR.

\section{References}

[1] Ali, R.M., Chu, N.E., Ravichandran, V. and Sivaprasad Kumar, S. First order differential subordination for functions associated with the lemniscate of Bernoulli, Taiwanese Journal of Mathematics, 16(3) (2012) 1017-1026.

[2] Janowski, W., Some extremal problems for certain families of analytic functions I, Ann. Polon. Math., 28 (1973) 297-326.

[3] Miller, S.S. and Mocanu, P.T., Differential subordination, theory and application, Marcel Dekker, Inc., New York, Basel, 2000.

[4] Omar, R. and Halim, S.A., Differential subordination properties of Sokol-Stankiewics starlike functions. Submitted. 
[5] Sokól, J. and Stankiewicz, J. Radius of convexity of some subclasses of strongly starlike functions, Folia Scient. Univ. Tech. Resoviensis,19 (1996) 101-105.

[6] Sokól, J., Radius problems in the class $S L^{\star}$, Applied Mathematics and Computation, 214 (2009) 569-573.

[7] Sokól, J., Coefficient estimates in a class of strongly starlike functions, Kyungpook Math. J., 49 (2009) 349-353.

[8] Sokól, J., On application of certain sufficient condition for starlikeness, J. Math. Appl., 30 (2008) 46-53

[9] Sokól, J., On sufficient condition to be in a certain subclass of starlike functions defined by subordination, Applied Mathematics and Computation, 190 (2007) 237-241. 